\title{
Nucleation, Mapping and Control of Cavitation for Drug Delivery
}

\author{
Eleanor Stride ${ }^{*}$ and Constantin Coussios \\ Institute of Biomedical Engineering, Department of Engineering Science, University of Oxford, Old \\ Road Campus Research Building, Oxford OX3 7DQ \\ *Email: eleanor.stride@eng.ox.ac.uk
}

\section{TOC Blurb:}

This Review describes how acoustic cavitation can be used to improve the delivery of drugs for the treatment of diseases such as cancer and stroke. Methods for seeding cavitation, treatment monitoring and current and future clinical applications are described.

\section{Key points:}

- A major challenge in the treatment of diseases such as cancer and stroke is achieving a sufficient concentration of a drug throughout the target region without producing toxic side effects elsewhere in the body.

- Oscillating microbubbles driven by ultrasound produce a range of mechanical, thermal and chemical effects that can be used to enable both localised delivery and improved distribution of drugs in tissue.

- This approach can be used to deliver both conventional small-molecule drugs and more recent biological therapeutics to areas of the body that are normally inaccessible, including across the blood-brain barrier and into solid tumours.

- Ultrasound-responsive microparticles and nanoparticles can either be used as drug carriers or co-administered with a free drug into the blood stream, providing cavitation nuclei that reduce the ultrasound pressures required to achieve effective drug delivery.

- The production of strong acoustic emissions during cavitation-enhanced delivery enables acoustic localization and mapping of bubble activity in real time for treatment monitoring.

\section{Abstract}

Acoustically driven bubbles produce a range of mechanical, thermal and chemical effects that can be exploited in drug delivery applications. Significant improvements in the targeting, distribution and efficacy of both current and emerging therapeutics can be achieved, from small molecules to biologics and nucleic-acid-based drugs. This Review describes how specially designed cavitation nuclei in the form of solid, liquid or gas particles can enable the triggered release of drugs, promote the permeabiliziation of challenging biological barriers and enhance drug delivery through tissue regions where diffusion alone is inadequate. Scalable strategies for mapping and controlling cavitation activity to harness its therapeutic potential at depth within the body are discussed, 
alongside current and emerging applications for the treatment of diseases including cancer and stroke.

\section{[H1] Introduction}

The term 'cavitation' encompasses bubble formation produced by changes in fluid pressure (hydrodynamic cavitation), heat (boiling), pressure waves (acoustic cavitation) and absorption of radiation (particle cavitation). Its usage also includes the dynamic behaviour of bubbles, in particular their expansion and collapse. Since the 19th century, cavitation has been investigated in fields as diverse as the hunting strategies of marine creatures ${ }^{1}$, accelerating chemical reaction ${ }^{2}$ and drug delivery ${ }^{3}$. Acoustic cavitation produced by ultrasound has been studied extensively in biological systems. Initially this was to establish standards for the safe use of ultrasound in medical applications $\mathrm{s}^{4,5}$. However, it was also recognized that cavitation could have beneficial effects, for example in the treatment of kidney stones using lithotripsy [G] ${ }^{6}$, in high-intensity focused ultrasound [G] (HIFU) surgery ${ }^{7}$, and in the intracellular delivery of molecules ${ }^{8}$.

The effects associated with cavitation are caused primarily by the dynamic behaviour of bubbles. Being filled with gas and/or vapour makes bubbles highly responsive to changes in pressure and temperature, both of which can cause rapid and dramatic changes in their volume. These volume changes lead to mechanical, thermal and chemical phenomena that can substantially impact their surroundings. In biological tissue, for example, cavitation may cause the destruction of malignant cells or the permeabilization of structures such as the blood-brain barrier ${ }^{9}$. These processes involve complex interactions between bubbles and the surrounding tissue, and are therefore challenging to predict. In this Review we focus on cavitation induced by exposure to an ultrasound field and its applications in drug delivery. We describe the mechanisms by which cavitation can be exploited to enhance the delivery, penetration and distribution of therapeutics into tissues. We discuss how cavitation is nucleated and monitored. We then survey current and potential applications of cavitation and propose future developments to enable clinical translation.

\section{[H1] Cavitation for drug delivery}

Successful drug delivery strategies maximize drug concentration at the target site or sites while minimizing off-target effects. Typically the target site is physically inaccessible, its location may not be precisely known or there may be multiple targets. Therefore, drugs are commonly delivered systemically via injection into the circulation, or via the digestive or respiratory tract. We use the term 'drug' to include both conventional small molecule drugs (mass $<1 \mathrm{kDa}$ and effective size $<5$ $\mathrm{nm}$ ) and more modern biological therapeutics such as antibodies (100-500 kDa; 10 nm), oligonucleotides (0.1-1 MDa; $<100 \mathrm{~nm}$ ), therapeutic viruses (> $10 \mathrm{MDa}$; 100-200 nm), or therapeutics reformulated as nanoparticles (1-1000 MDa; 50-500 nm).

Once introduced into the bloodstream, a drug is transported throughout the body. The quantity delivered to a target site is limited by several factors (Fig. 1). The toxicity of the drug restricts its maximum safe blood concentration, to avoid damage to healthy tissue. The circulation time is limited; that is, a drug is only active for a finite time because of degradation and/or clearance from the blood stream. Unless the target is vascular [G], the drug must diffuse out of the vessel across the endothelium [G] and through the surrounding tissue. The larger and/or more highly charged the drug, the lower its diffusivity - a particular challenge for large proteins or viral particles. In a malignant tumour, diffusion may be further restricted by elevated pressure due to reduced lymphatic drainage and an increase in tissue density due to rapidly proliferating cells. Once at the 
target site, the drug must be taken up by the target cells. Additionally, in some cases the drug must also reach a specific cellular compartment, such as the nucleus, to be effective. The next sections describe how cavitation can overcome these barriers.

\section{[H2] Triggered release and activation}

Encapsulating a drug within a carrier such as a liposome or polymeric nanoparticle can protect it from degradation, limit off-target interactions and increase its circulation time ${ }^{10}$. It is desirable, however, to be able to release the drug 'on demand'. Cavitation can be used to trigger release from particles and/or activation of drugs through several mechanisms ${ }^{11,12,13}$ (Fig. 2).

\section{[H3] Mechanical effects}

When exposed to an ultrasound field, bubbles undergo volumetric oscillations, owing to the timevarying pressure. For therapeutic applications, the field typically will have a centre frequency of $0.5-$ $2 \mathrm{MHz}$ and a peak negative pressure amplitude of $0.5-5 \mathrm{MPa}$. Even at moderate amplitudes $<500$ $\mathrm{kPa}$ at $1 \mathrm{MHz}$ ), the acceleration of the bubble wall may be sufficient to impose significant forces upon nearby surfaces that can be used to promote drug release from particles, depending on their size and mechanical rigidity. Momentum transfer from the oscillating bubble to the surrounding liquid through interfacial friction can also produce localized flows known as microstreaming [G] ${ }^{14}$ (Fig. 2). If the bubble is close to a surface, such as a blood vessel wall or another bubble, it can exhibit highly non-spherical oscillations ${ }^{15,16,17}$ giving rise to microstreaming velocities on the order of $1 \mathrm{~mm} \mathrm{~s}^{-1}$ (Ref. ${ }^{18}$ ). This motion in turn imposes shear stresses upon any nearby structures, again potentially enabling drug release.

Another phenomenon associated with non-spherical bubble oscillations near a surface is the generation of a liquid microjet during bubble collapse ${ }^{43}$. If there is sufficient asymmetry in the acceleration of the fluid on either side of the collapsing bubble, then the more rapidly moving fluid may deform the bubble into a toroidal shape, causing emission of a high-velocity jet on the opposite side (Fig. 2). Microjetting is well known to cause pitting even in highly resilient materials such as steel ${ }^{19,20}$, and similar damage to solid particles has also been observed and correlated with drug release ${ }^{21}$. Microjetting is typically seen at relatively low frequencies $(<500 \mathrm{kHz})$ and/or high pressures ( $>1 \mathrm{MPa}$ at $1 \mathrm{MHz}$ ). In this regime, bubbles may also collapse such that the bubble wall velocity becomes supersonic. The supersonic motion of the bubble wall creates a discontinuity in the pressure in the surrounding liquid, leading to the emission of a shockwave and large stresses on nearby structures. Like microjets, shockwaves have also been correlated with particle disruption ${ }^{22}$.

Even at very low amplitudes ( $<0.1 \mathrm{MPa}$ at $1 \mathrm{MHz}$ ), oscillating bubbles reradiate energy from the incident ultrasound field in the form of spherical pressure waves. Owing to the compressibility of bubbles, the amplitude of these waves is considerably larger than the acoustic scattering from a rigid sphere of equivalent size ${ }^{23}$. Additionally, the nonlinear character of the microbubble oscillations leads to energy being reradiated over a range of frequencies. At moderate driving pressures $(<0.5$ $\mathrm{MPa}$ ) the bubble spectrum contains integer multiples (harmonics) of the driving frequency; at higher pressures ( $>0.5 \mathrm{MPa}$ ) also fractional components (subharmonics and ultraharmonics). As discussed below, this is the reason for the widespread use of microbubbles as ultrasound contrast agents: the harmonic emissions are readily distinguished from the linear echoes from surrounding tissue, and can also be exploited for treatment monitoring in drug delivery.

\section{[H3] Thermal effects}

The nonlinear acoustic radiation from oscillating bubbles also leads to heating of the immediate surroundings. In biological tissue, absorption of ultrasound increases with frequency. Thus, because 
of the higher harmonics in the radiation from the bubbles, quite dramatic increases in temperature can be produced by cavitation ${ }^{24,25,26}$. This effect can enhance thermal ablation and release drugs from thermally sensitive carriers ${ }^{27}$. Bubbles also dissipate energy because of viscous friction in the liquid and thermal conduction from the gas core, the temperature of which increases during compression. Which mechanism is dominant depends on the size of bubble, the driving conditions and the viscosity of the medium ${ }^{48,49,28}$. However, conductive heating is typically negligible in biomedical applications of ultrasound because the time constant of heat transfer is much longer than the period of the microbubble oscillations ${ }^{29}$.

\section{[H3] Chemical effects}

Although sufficient to promote drug release, the temperature rise produced in the surrounding tissue is negligible compared to that occurring inside the bubble, where it may reach several thousand Kelvin during a rapid bubble compression ${ }^{30}$. The gas pressure similarly increases substantially during this time. These extreme conditions are only sustained for a brief period $(<1 \mu \mathrm{s})$, but can lead to emission of electromagnetic radiation (sonoluminescence) and production of highly reactive chemical species, in particular reactive oxygen species (ROS). ROS are important for multiple biological processes ${ }^{31,54}$ and substantial quantities can be generated in the surrounding fluid by cavitation under conditions relevant for drug delivery (<1 MPa peak negative pressure at $1 \mathrm{MHz}$ ), ${ }^{32}$. The importance of ROS generation and/or sonoluminescence in terms of their direct impact on cells has yet to be determined because direct measurements of these effects in tissue are challenging.

Another question is whether there are any deleterious effects on the drugs themselves. To the best of our knowledge no studies have indicated degradation of drugs by cavitation under mild therapeutic conditions; even highly sensitive drugs such as small interfering ribonucleic acids (siRNA) are unaffected ${ }^{33}$. Indeed, the chemical effects of cavitation can be used to activate certain types of drug, a phenomenon termed sonodynamic therapy ${ }^{34}$. This enables treatment localization because toxic species are confined to the ultrasound focus and off-target toxicity is negligible.

\section{[H2] Cavitation-enhanced transport}

Controlling the release and/or activation of a drug improves targeting, but is no guarantee of effective delivery. A sufficient concentration must also reach the target cells. Exposure to focused ultrasound of drugs co-administered with microbubbles promotes their transport out of the blood stream ${ }^{35}$ and into challenging biological targets such as solid tumours where the elevated interstitial pressure and dense extracellular matrix hinder the penetration of drugs ${ }^{36}$. Transport of drugs has been reported over length scales of $\mathrm{mm}$ in tissue phantoms [G] ${ }^{37}$ and hundreds of micrometres in vivo ${ }^{38}$. The detailed mechanisms are not yet fully understood, and multiple phenomena are relevant.

First, transport of drug molecules increases as a result of the convective flow produced by microstreaming around an oscillating bubble ${ }^{39}$. Second, opening of pores and channels within and between endothelial cells can be stimulated by the shear stresses generated by microstreaming ${ }^{17}$ and/or direct impingement by bubbles ${ }^{40,41}$. This opening facilitates transport of drugs into extravascular tissue either directly or via transcytosis [G] - particularly relevant in the brain where the tightly packed layer of endothelial cells, the blood-brain barrier (BBB), represents a significant challenge to drug delivery ${ }^{42}$. Third, there is evidence that ROS and other chemical species may induce intercellular and intracellular transport ${ }^{43} 44$. 
These mechanisms are associated with relatively mild exposure conditions $(<1 \mathrm{MPa}$ peak negative pressure at $1 \mathrm{MHz}$ ) and may involve both direct mechanical effects and stimulation of biological responses ${ }^{46}$. At higher pressures, microjetting and ballistic motion of bubbles may create larger openings in the vessel wall and surrounding tissue. Ballistic motion results from the pressure gradient in the fluid generated by a propagating ultrasound wave (primary radiation force). Owing to their high compressibility, bubbles can travel at high velocities, sufficient to penetrate biological tissue. Bubble 'tunnelling' has been reported ${ }^{47,48,49,50}$ and may explain why vessel permeabilization appears to be reversible at low ultrasound intensities but correlated with permanent damage under more intense exposures ${ }^{51}$.

However, interactions between bubbles and tissue are far from fully understood and there are two aspects in particular that require further investigation, discussed here.

\section{[H3] Bubble-boundary interactions}

The behaviour of a bubble close to a surface can be extremely complex ${ }^{52}$, including phenomena such as asymmetric oscillations, microstreaming and, at sufficiently high pressures, microjetting. Furthermore, the interaction between the reradiated ultrasound field and its reflection from the surface leads to a pressure gradient in the liquid and a secondary radiation force [G] on the bubble. The elastic properties of the boundary determine the phase difference between the radiated and reflected waves and hence whether the bubble moves towards or away from the surface. These properties similarly determine the direction of any microjetting. Additionally, non-spherical bubble dynamics may be important for the release of drugs during delivery from coated microbubbles ${ }^{53}$. Unfortunately, the viscoelastic and heterogenous nature of tissue hinders prediction of the behaviour of bubbles in vivo and observations made in vitro in conventional cell culture chambers may be of limited value ${ }^{54}$. Moreover, in many cases a bubble is confined in multiple directions, for example in a narrow capillary ${ }^{55}$, and the dynamics of the system (bubble, vessel and surrounding tissue) as a whole must be considered ${ }^{56}$.

\section{[H3] Bubble-bubble interactions}

We have discussed cavitation phenomena in the context of a single bubble for the sake of simplicity. However, in drug delivery applications the observable effects of cavitation are typically produced by a population of bubbles and the influence of inter-bubble interactions should not be ignored. Secondary radiation forces are generated between oscillating bubbles, inducing the formation of large clusters ${ }^{57}$ that may behave very differently from individual bubbles. For example, bubble clusters experience much larger primary radiation forces and this may explain why the diameter of the 'tunnels' observed experimentally is much larger than that of the bubbles present ${ }^{47}$.

\section{[H2] Sonoporation and intracellular transport}

At the cellular level, cavitation-mediated permeabilization has been widely observed in cases where bubbles and cells are in close proximity. Similar mechanisms to those described above are thought to be responsible; we refer readers to earlier reviews ${ }^{58,59}$ for a detailed discussion. There is some evidence that intracellular streaming also occurs, which may promote transport within the cytoplasm ${ }^{18}$. 


\section{[H1] Cavitation Nucleation}

\section{[H2] Ultrasound-mediated nucleation}

In a pure liquid, whether it is thermodynamically favourable for a bubble to exist is determined by the local pressure and temperature. This is because temperature and pressure govern the state of the medium, the maximum concentration of dissolved gas and the size of any pre-existing bubbles.

For a bubble to form, energy must be provided to create a new surface. According to classical nucleation theory, this energy is that required to overcome intermolecular bonds, that is, the tensile strength of the liquid. In water, such homogenous nucleation would require negative pressures of the order of $10^{8} \mathrm{~Pa}$ and yet, even in pure water, bubbles form following much smaller pressure changes and in the absence of heating. In the 1940s it was proposed that this discrepancy could be explained by the presence of discontinuities, that is, places where the medium structure is already disrupted, enabling bubbles to form more readily via heterogenous nucleation ${ }^{60}$.

This proposal was made in response to the problem of bubble formation in tissue, but the hypothesis is equally applicable to simple liquids. Indeed it was subsequently demonstrated that the pressure required to generate bubbles in water was directly related to its purity ${ }^{61}$, evidencing the role of discontinuities as bubble nucleation sites. The exact nature of these sites is still the topic of some debate ${ }^{62}$, but the most widely accepted theory - the crevice model - is that bubbles originate from hydrophobic crevices or pores in which gas is trapped. These crevices may be located on either the surfaces bounding the liquid or microscopic particles within it ${ }^{63,64,65}$. There is also strong evidence to suggest that many liquids contain microscopic and nanoscopic bubbles stabilized by surfactants ${ }^{66,67}$ that may also act as nuclei - the skin model (Fig.3).

Cavitation nucleation by ultrasound in tissue and by quasi-static pressure reduction in a pure liquid occur by fundamentally the same process. However, the time dependence of both temperature and pressure need to be considered. As ultrasound propagates through a medium, the pressure varies periodically at the frequency of the incident wave. The temperature may also increase as a result of absorption. If the intensity of the ultrasound is sufficient, such as in HIFU surgery, then the temperature rise may create vapour bubbles - that is, boiling occurs. However, at moderate intensities the temperature rise is small. In that case, bubble formation depends on whether the peak negative pressure of the ultrasound is sufficient to allow gas trapped at a nucleation site to expand. This expansion occurs against the combined constraints of the inertia and viscosity of the surrounding liquid and the surface tension of the bubble, which arises from the gas-liquid interfacial tension and any elastic resistance presented by a surfactant coating. At very low frequencies, surface tension is the dominant factor and the pressure required for bubble formation is known as the Blake pressure ${ }^{68}$. The smaller the nuclei or the higher the surface tension, the larger this pressure is. At the $\mathrm{MHz}$ frequencies relevant for therapeutic applications, bubble growth is further restricted by liquid inertia and viscosity. There may be insufficient time during the negative cycle of the ultrasound pulse for the bubble to expand enough to produce a measurable effect. Thus, the pressure required for nucleation increases with increasing driving frequency. The duration of ultrasound exposure is also important, because bubble nuclei can grow over several cycles through a process known as rectified diffusion ${ }^{69}$. Hence the pressure required for nucleation may decrease with increasing pulse length or duty cycle.

\section{[H2] Cavitation agents}


A significant advantage of ultrasound is that it can be tightly focused, offering a high degree of control over when and where cavitation occurs. However, the pressure amplitudes required to activate endogenous nuclei, that is those naturally present in tissue, are relatively high and vary considerably with location and between subjects. As in pure liquids, the exact nature of nuclei in tissue is unknown but a plethora of particles, inhomogenous surfaces and surfactant molecules is present. To address this variation, exogenous or artificial cavitation nuclei are introduced. When a known concentration of nuclei with a known acoustic response are present at a target site, bubbles can be generated more reproducibly at lower pressures. The cavitation agents that have been investigated for drug delivery applications are broadly grouped into three classes (Fig.3) discussed here.

\section{[H3] Gas bubbles}

The most widely studied nuclei to date have been gas microbubbles stabilized by a surfactant or polymer coating. Although clinically approved as contrast agents for diagnostic ultrasound applications, they have been shown to enhance multiple types of ultrasound therapy in animal studies and also clinically (discussed below). Contrast agent microbubbles typically have diameters of $1-10 \mu \mathrm{m}$. At room temperature $\left(21^{\circ} \mathrm{C}\right)$ and pressure ( 1 atmosphere) contrast agent microbubbles have mean diameters of $\sim 2 \mu \mathrm{m}$, however, at physiological temperature $\left(37^{\circ} \mathrm{C}\right)$ bubbles expand. For clinically relevant ultrasound frequencies of $0.5-2 \mathrm{MHz}$, the pressures required to produce therapeutically relevant amplitudes of oscillation are typically less than $1 \mathrm{MPa}$, that is, considerably below those required to stimulate naturally occurring nuclei. The microbubble coating both reduces surface tension and provides a barrier to gas diffusion. Depending on its thickness and viscoelastic properties it may also reduce the amplitude of oscillation at a given pressure and increase the mechanical resonant frequency of the bubble ${ }^{70}$. Additionally, the coating material can play a direct role in drug delivery by modifying the permeability of cells through transfer of material prior to ultrasound exposure ${ }^{71}$.

In most studies, microbubbles have been co-injected systemically with a drug. However, the coating can be further exploited to improve treatment localization by providing a substrate to which drug molecules are bound. The method of attachment depends on the nature of the drug ${ }^{72,73}$. For example, hydrophobic drugs may be encapsulated between the gas core and the coating, whereas hydrophilic drugs may be conjugated to the outer surface either directly ${ }^{74}$ or in the form of liposomes ${ }^{75}$. In addition to reducing systemic toxicity, conjugation improves cellular uptake ${ }^{76}$. Other molecules may also be attached to the bubble surface to facilitate targeting to a specific site. Multiple approaches have been investigated in the contexts of drug delivery and molecular imaging. One is to use antibodies to enable binding at the endothelium, such as anti-vascular endothelial growth factor (anti-VEGF) which enables binding at sites of endothelial cell proliferation or antivascular cell adhesion molecule (anti-VCAM) which enables binding at sites of inflammation. Another approach is incorporation of magnetic nanoparticles to enable concentration of microbubbles at a target by an applied magnetic field ${ }^{77,78}$. Current clinical contrast agents contain gas with high molecular weight and low solubility, such as perfluoropropane or sulphur hexafluoride. However, microbubbles are also being investigated for the delivery of therapeutic gases, such as oxygen to improve the response of hypoxic tumours ${ }^{79}$, and nitric oxide for cardiovascular disease ${ }^{80}$.

The echogenicity [G] of microbubbles that makes them excellent contrast agents is also useful for drug delivery because it enables them to be tracked through the circulation using low-intensity ultrasound. However, microbubbles do have several disadvantages. They are too large to extravasate prior to ultrasound exposure. Additionally, owing to their size, the circulation half-life of microbubbles is relatively short ( $<5 \mathrm{mins})$, restricting the treatment window ${ }^{81}$. They are also rapidly destroyed once exposed to ultrasound which limits their ability to promote the activation and transport effects described above. 


\section{[H3] Liquid droplets}

One approach to address some of the limitations of microbubbles is to form gas bubbles using submicrometre sized droplets of volatile liquids that vaporize upon exposure to ultrasound ${ }^{82}$. The boiling point of the liquid, typically a perfluorocarbon, is sufficiently low for it to exist in a gaseous state at $37^{\circ} \mathrm{C}$. However, the size of the droplet gives rise to a high surface pressure that maintains the core in a superheated liquid state. Once exposed to ultrasound, the droplets form gas microbubbles, which behave as described above. The small size of droplets enables them to circulate in the blood stream for tens of minutes prior to ultrasound exposure, thus increasing the treatment window and improving their targeting potential compared with microbubbles. It may also allow them to extravasate in the 'leaky' vasculature associated with cancerous tumours ${ }^{82}$, enabling cavitation activity at greater tissue depths. However, the clinical utility of the so-called enhanced permeation and retention effect has been questioned ${ }^{83}$.

Liquid droplets can be coated with similar materials to those used for gas microbubbles for stabilization and attachment of functional species $33,82,84,85,86,87$, and have been investigated in most of the same applications. In vivo, liquid droplets have been reported as providing better control over lesion volume for thermal ablation ${ }^{88}$ compared with microbubbles. Other studies have demonstrated improved distribution of chemotherapy within a tumour ${ }^{89}$ and more homogenous delivery of a model drug across the BBB ${ }^{90}$.

Liquid droplets have some disadvantages compared to microbubbles. Droplets typically require higher pressures to initiate vaporization, although this does depend on the perfluorocarbon and coating material used. Unlike microbubbles, droplets cannot be imaged with ultrasound prior to vaporisation. Furthermore, their acoustic response is currently less well understood. In particular, there is a complex relationship between droplet size, ultrasound frequency, pulse length, duty cycle and the pressure required to produce vaporisation ${ }^{91}$. The inverse relationship between vaporization pressure and driving frequency observed with larger (micrometre scale) droplets is due to superharmonic focusing [G] ${ }^{92}$. However, this cannot explain the nonlinear relationships observed for some formulations of sub-micrometre droplets.

Finally, gaseous 'nanobubbles' with diameter $<1 \mu \mathrm{m}$ have also been studied. These offer the advantages of droplets in terms of circulatory stability and extravasation [G] potential, but respond at much lower ultrasound pressures than droplets do ${ }^{93}$. However, there is some controversy about the mechanism of cavitation enhancement ${ }^{94}$.

\section{[H3] Solid particles}

Another type of cavitation agent consists of solid particles containing hydrophobic cavities which entrap gas, thereby providing nucleation sites. Like droplets, such particles can be produced with sizes that facilitate favourable circulation times and extravasation potential. Several types of biocompatible particles have been investigated including mesoporous silica ${ }^{95}$, carbon nanotubes ${ }^{96}$, polymer 'sponges' ${ }^{97}$, polymeric 'cups' ${ }^{98}$ and gold cones ${ }^{99}$. Particles that generate gas bubbles through chemical reaction have also been tested ${ }^{100}$.

A major advantage of solid particles over gas bubbles and liquid droplets is the degree to which solid particles can sustain cavitation, owing to the fact that the particles are not destroyed by exposure to ultrasound. Thus, the particles can continuously nucleate bubbles, provided the hydrophobic cavities do not become wetted. The combination of size and stability also enables solid particles to promote cavitation activity at greater tissue depths. Like droplets, solid particles require higher pressures than microbubbles for activation and they cannot be imaged prior to activation. It is also challenging 
to functionalize solid particles for targeting or drug loading, because of the risk of wetting the hydrophobic cavity. Moreover, certain types of particle suffer from safety concerns, such as the potential of mesoporous silica to induce thrombosis, and the possible carcinogenicity of carbon nanotubes ${ }^{101}$.

\section{[H1] Cavitation Monitoring}

\section{[H2] Classifying cavitation}

\section{[H3] A cautionary note on cavitation thresholds}

How cavitation is classified depends on the phenomena of interest. These include bubble nucleation, acoustic emissions, sonoluminescence or the likelihood of mechanical damage. Consequently there are multiple "thresholds" that can be defined to characterise different types of bubble behaviour. The most commonly used in drug delivery applications is the inertial cavitation threshold. It is important, however, to understand the physical meaning of this threshold and whether or not it is relevant for a given application.

Numerous mathematical formulations exist to analyze bubble dynamics ${ }^{102}$. The motion of the bubble is primarily determined by the competition between the liquid inertia and the internal gas pressure. This competition can be characterized ${ }^{103}$ by comparing the relative contributions of inertia and pressure to the acceleration of the bubble wall, using the Rayleigh-Plesset equation

where the inertial factor

$$
\frac{\mathrm{d}^{2} R}{\mathrm{~d} t^{2}}=\mathrm{IF}+\mathrm{PF}
$$

and the pressure factor

$$
\mathrm{IF}=-\frac{3}{2 R}\left(\frac{\mathrm{d} R}{\mathrm{~d} t}\right)^{2}
$$

$$
\mathrm{PF}=\left(\frac{p_{G}(R)+p_{\infty}(t)-2 \sigma / R}{\rho_{L} R}\right) .
$$

$R$ is the time-dependent bubble radius with initial value $R_{o}, p_{G}$ is the pressure of the gas inside the bubble, $p_{\infty}$ is the combined hydrostatic and time-varying pressure of the liquid, $\sigma$ is the surface tension at the gas-liquid interface and $\rho_{L}$ is the liquid density. Various forms of the Rayleigh-Plesset equation have been studied and several more complex equations of motion have been derived that incorporate factors such as liquid viscosity and compressibility or thermal effects. For more information on these models, we refer readers to Ref. ${ }^{102}$

Two scenarios arise, depending on which factor dominates when the bubble approaches its minimum size. In the non-inertial scenario, the pressure factor is dominant and the bubble undergoes sustained volume oscillations. If the inertia term is dominant, the bubble undergoes inertial collapse, after which it may either rebound or disintegrate. Which of these scenarios occurs depends on the bubble expansion ratio $R_{\max } / R_{o}$, where $R_{\max }$ is the maximum radius of the bubble, which in turn depends on the amplitude and frequency of the applied ultrasound field. This analysis, developed in Ref. ${ }^{103}$, provides a framework for categorizing cavitation behaviour in terms of the violence of bubble collapse, in other words, whether the cavitation is inertial or non-inertial. From 
this a threshold for inertial cavitation can be defined in terms of either a critical pressure amplitude for a given bubble size and driving frequency, or a critical bubble size for a given pressure amplitude. We note that historically the term "stable cavitation" was used to describe prolonged smallamplitude bubble oscillations, and "transient cavitation" described rapid violent bubble collapse, which is more likely to produce damage. However, the term stability may refer to the periodicity of bubble oscillations, bubble persistence, or the consistency of secondary effects such as acoustic emissions. Moreover, it is possible for a gas bubble to undergo periodic violent collapses ${ }^{104}$.

Alternative criteria for categorizing bubble behaviour include the interior temperature exceeding a threshold and the bubble wall velocity exceeding the speed of sound in the liquid ${ }^{105}$. The Blake pressure defined above similarly provides the basis for defining a limiting threshold for bubble nucleation. These analyses have been used to define the mechanical index, a conservative safety index for use in diagnostic applications that determines the likelihood of an inertially collapsing bubble being created from a pre-existing gaseous nucleus in response to a single cycle of ultrasound of a given frequency and amplitude ${ }^{106}$.

Equivalent standards do not yet exist for therapeutic applications, and the definition of an appropriate cavitation threshold (or thresholds) for drug delivery poses several challenges. First, as mentioned above, cavitation typically involves clouds of bubbles arising from heterogeneous populations of nucleation sites and thus there exists a distribution of thresholds both for nucleation and inertial cavitation. Therefore, it is often more practical to consider cavitation events in terms of probability: for a given driving frequency, the threshold pressure corresponds to a percentage probability of the relevant cavitation event. Second, exactly what constitutes a relevant cavitation event is difficult to define, owing to the wide range of phenomena discussed above, whose importance may vary considerably between applications. Third, to be useful, a threshold should be related to a quantity that can be monitored during treatment.

\section{[H3] Cavitation emissions}

Several methods exist for measuring cavitation activity, but for real-time monitoring that can be translated into clinical applications, acoustic methods are usually the most practical because they can be implemented non-invasively using external probes. These methods exploit the secondary sound fields radiated by oscillating or collapsing bubbles. As discussed above, even at relatively low amplitudes, bubbles behave in a highly nonlinear fashion and their emissions contain multiples (harmonics) of the driving frequency. As the pressure increases, so does the range of frequencies in the emission spectrum, which includes fractional harmonics and, at high enough pressures, broadband noise. Thus, both the amplitude and frequency content of the cavitation signal indicate the behaviour of the bubbles: a sparse population of bubbles at low amplitudes produces predominantly harmonic emissions; more energetic bubble activity produces broadband noise. However, cavitation signals must be interpreted with care. Harmonics are also produced by nonlinear propagation and subsequent linear scattering of the incident field. The range of bubble sizes continually changes and this contributes to broadband noise. The bandwidth and resolution of the electronic components in the detection system also determine the characteristics of the recorded spectrum. There is no simple one-to-one mapping between bubble dynamics and acoustic emissions, which should always be interpreted based on adequate prior knowledge of the acoustic field, nucleation environment and detector characteristics.

\section{[H2] Active detection}


Acoustic emissions are detected either actively (pulse-echo mode) or passively (emission detection) 107. Active detection uses the same principles as ultrasound imaging or sonar. Pulses of ultrasound are transmitted into a tissue volume and the amplitude and time delay associated with the backscattered signals provide information about the concentration and scattering strength of scatterers present. Because bubbles are strong scatterers of ultrasound (strictly speaking, reradiators), they produce large signals and are readily detected. The strength and harmonic content of the signal can be used to infer the size and concentration (number of bubbles per unit volume) of bubbles present and their dynamic behaviour, subject to the caveats above.

The simplest method uses a single-element transducer, separate from the therapy transducer, whose bandwidth coincides with a suitable range of higher harmonics of the driving field. The detecting transducer may be fixed to focus on a single volume of tissue, a process known as A-mode imaging, or it may be scanned across the region of interest. More commonly, instead of mechanically scanning, a transducer array is used to interrogate the region of interest. The ultrasound beam is swept electronically across the region and the echo time used to isolate scattered signals corresponding to a particular tissue depth (time gating). The amplitude of these signals is used to produce a greyscale image, with the intensity of each pixel corresponding to the magnitude of scattering from that area of the imaging plane. This process, known as B-mode imaging, is the same process as used in conventional ultrasound diagnostic imaging and treatment monitoring in ultrasound-guided therapy systems.

Bubbles, particularly the large vapour-filled bubbles produced during HIFU surgery, are readily detected using B-mode ultrasound imaging ${ }^{108,109}$. Indeed, their size often poses a challenge for treatment monitoring because their very high echogenicity causes shadowing of the underlying tissue and changes to the speed of sound that distort the image formed. More sensitive detection of smaller microbubbles can be achieved by exploiting their highly nonlinear behaviour and the same techniques used for contrast-enhanced ultrasound imaging can be used for cavitation monitoring. A drawback of these approaches is that they require the monitoring transducer to have a large bandwidth, which increases cost and/or restricts sensitivity. Furthermore, the need to transmit pulse sequences reduces the temporal resolution of the monitoring system and there may be signal distortion due to nonlinear propagation that limits the signal-to-noise ratio. Some of these limitations are overcome by ultrafast active imaging approaches ${ }^{110,111 .}$

\section{[H2] Passive detection}

A major disadvantage of active detection methods is that therapeutic signals have high amplitudes, and therefore cannot be transmitted simultaneously with imaging or monitoring signals because of saturation of the detector; instead the signals must be alternated ${ }^{108,112}$. This both complicates the system design and reduces flexibility in the therapeutic protocol. Because cavitation bubbles produce acoustic emissions in response to the therapeutic signal, however, a separate imaging signal is unnecessary. Instead, monitoring devices can detect cavitation passively. The simplest approach is to use a passive cavitation detector, which is a single-element transducer focused on the region of interest. This method enables monitoring over time of the formation and activity of bubbles. Passive methods can be far more sensitive than active methods for detecting inertial cavitation in particular. This is because an inertially cavitating bubble has a measurable scattering cross-section for periods of the order of $n s$, much smaller than the time typically required to form an active image ${ }^{109}$, and thus may be 'missed'.

As is done for active methods, arrays of transducers can be used for passive monitoring to detect and beamform [G] the acoustic emissions from tissue volumes into a spatial map of cavitation 
activity ${ }^{113}$. Instead of using echo times as in active imaging, this passive acoustic mapping (PAM) localizes sources of cavitation through source coherence metrics ${ }^{114}$. Beamforming is performed in either the time domain ${ }^{113,114}$ or the frequency domain ${ }^{115,116,117}$. Whereas pulse-echo techniques use short-duration broadband excitation signals, PAM exploits the narrowband nature of the signals that cause the cavitation activity, and can thus achieve far better spectral resolution, enabling classification of cavitation activity in real time ${ }^{118}$ and beamforming of narrowband and broadband emissions separately ${ }^{119}$.

A disadvantage of PAM is that its axial resolution is not a function of the transmitted pulse length, but of the point-spread function that depends on the array geometry, and the pulse centre frequency and bandwidth. The point-spread function describes how a point source appears after broadening as a result of diffraction, and thus determines the minimum distance between cavitation events that is resolved using a particular receiver array. Additional beamforming challenges include the need to deal with spherical spreading of the acoustic emissions from the source to the receiver; to account for uncertainties in the speed of sound and attenuation of the intervening medium; and to correct for the diffraction patterns of individual array elements that in reality are not true point receivers ${ }^{120}$. More robust passive beamforming techniques based on the Capon beamformer improve the axial resolution of PAM, reduce artefactual interference signals arising from multiple scattering between bubbles, and improve resilience to array elements of varying sensitivities ${ }^{121}$. PAM techniques can be implemented in real-time and combined with active imaging techniques on the same ultrasound array to provide a complete system for guidance and monitoring of cavitationmediated drug delivery ${ }^{122,123}$.

\section{[H1] Applications}

Cavitation in tissue can mediate a wide range of therapeutically beneficial ${ }^{28}$ or potentially damaging ${ }^{124}$ bioeffects, which depend on the type of cavitation nucleation agent used, the ultrasound frequency and amplitude, the duty cycle, the ratio of vessel diameter to bubble size at the target site and the surrounding tissue properties. As a method that is uniquely capable of enabling local enhancements in transport and permeability, cavitation-mediated drug delivery is increasingly being applied to facilitate transport of therapeutics across some of the most biologically inaccessible barriers, including the stromal layer [G] in solid tumours, the BBB, the spinal cord, occluded vessels in stroke, heart muscle and skin (Fig.4).

\section{[H2] Drug Delivery for Cancer}

Despite their leaky vasculature ${ }^{125}$, solid tumours present a formidable barrier to drug delivery due to their elevated interstitial pressure, dense extracellular matrix and sparse vascularity ${ }^{36}$. This makes diffusion-driven delivery from the bloodstream into tissue highly ineffective, not least because live cancer cells in tumours can reside almost twice the distance $(180 \mu \mathrm{m})$ from blood vessels than healthy cells $(100 \mu \mathrm{m})$. Convective transport mediated by cavitation from artificial nuclei enables the penetration of large therapeutics over distances of hundreds of micrometres ${ }^{38}$ both in vitro ${ }^{126}$ and in vivo ${ }^{127}$ (Fig. 4a). Enhancements in drug delivery have also been achieved with inertial cavitation from endogenous nuclei ${ }^{128,129,130}$, but at the cost of microvascular rupture inside the tumour and extensive haemorrhage in treated areas. Cavitation agents provide a safer and more effective method of intratumoral drug delivery. In this context, solid gas-stabilizing nuclei that can penetrate the leaky tumour vasculature exhibit substantially enhanced intratumoral delivery and penetration compared to microbubbles ${ }^{131}$. In certain tumour types, such as in pancreatic cancer, delivery is 
further hindered by the presence of a thick, impenetrable stromal layer between blood vessels and the target tumour cells ${ }^{130}$. A clinical report suggests that microbubble-mediated cavitation improved drug delivery to pancreatic tumours ${ }^{132}$ but further randomized studies in larger patient cohorts are required to verify these findings.

\section{[H2] Blood Brain Barrier Permeabilization}

Another formidable barrier to drug delivery is the BBB. This arrangement of tight junctions between the cells lining the vessels in the brain prevents toxic species from reaching the central nervous system but also hinders transport of therapeutic molecules larger than $500 \mathrm{Da}$ to treat conditions such as cancer, Alzheimer's disease and Parkinson's disease. In 2001, ultrasound-mediated cavitation was reported to enable reversible opening of the BBB ${ }^{133}$. Later studies demonstrated that inertial cavitation produced undesirable erythrocyte [G] extravasation and cell death whereas non-inertial cavitation appeared to mediate safe and reversible BBB opening for drug delivery ${ }^{134}{ }^{135}$. A wide range of timescales for the duration of $\mathrm{BBB}$ opening has been reported depending on the measure used, probably on account of the multiple mechanisms involved as discussed above. Therefore, a key challenge is treatment monitoring; recent developments have indicated that adaptations of PAM ${ }^{113}$, ${ }^{114,118}$ can provide an invaluable tool for real-time $3 \mathrm{D}$ transcranial treatment monitoring ${ }^{136,137}$.

Two pioneering approaches enable clinical translation of cavitation-mediated reversible BBB opening. The first uses a locally implanted radial probe inserted through a small perforation in the skull to provide highly spatially localized ultrasonic excitation; a systemic infusion of microbubbles with the relevant drug or drugs is given simultaneously (Fig. 4c). Initial results in ten patients have demonstrated safe and effective reversible opening of the BBB, but also acknowledged limitations of this approach in terms of the depth and location of addressable sites in the brain ${ }^{138}$. A second noninvasive and potentially more versatile approach uses a transcranial array and active beamforming approaches to focus ultrasound through the skull. This system was recently successfully tested in humans ${ }^{139}$ and further trials are underway for the treatment of Alzheimer's disease and cancer.

\section{[H2] Cardiovascular Applications}

Another active area of research is sonothrombolysis, the breakdown of blood clots using ultrasound, for ischaemic [G] stroke therapy ${ }^{140}$. In this case, the main role of cavitation is to facilitate the transport and penetration of recombinant tissue plasminogen activator along partially occluded vessels and into dense and often calcified blood clots ${ }^{141}$ to dissolve them and restore blood flow as soon as possible. Early studies with microbubbles indicated that microstreaming associated with non-inertial cavitation enhanced thrombolysis ${ }^{142,143}$; more recent studies have also suggested acoustic radiation forces acting on the microbubbles as a contributing mechanism ${ }^{144}$. The few clinical studies of microbubble-enhanced sonothrombolysis to date have provided predominantly encouraging results in small numbers of patients, evidencing early recanalization [G] and the possibility of improved neurological outcomes for patients with ischaemic stroke ${ }^{145}$, and faster rates of epicardial [G] recanalization and reduced left ventricular remodelling in heart attack patients ${ }^{146,}$ 147 (Fig.4d). Unfortunately, however, there have been some notable and tragic exceptions. For example, in the Transcranial Ultrasound in Clinical Sonothrombolysis (TUCSON) trial ${ }^{148}$ there were two patient fatalities whose cause is as yet unexplained. Therefore, clinical translation of this promising treatment requires better mechanistic understanding, the incorporation of improved techniques for targeting cavitation-enhanced sonothrombolysis ${ }^{149}$, and real-time treatment monitoring, for example by passive acoustic mapping of cavitation activity $117,136,150,151$. 


\section{[H2] Drug Delivery to the Spinal Cord}

The blood-spinal cord barrier (BSCB) presents a challenge for the delivery of therapeutics to the spinal cord, similarly to the BBB. The use of ultrasound in the spine has traditionally been considered unfeasible, owing to the difficulties in transmission and focussing through the complex and highly attenuative bone geometry. However, studies have indicated that such targeted therapy is possible in vertebral bodies ${ }^{152,153}$, and that reversible opening of the BSCB was possible following systemic administration of microbubbles ${ }^{154}$. These findings could enable improved treatment of leptomeningeal metastases - a serious complication of cancer in the central nervous system - and ultimately the technique could be extended to enable repair and regeneration of the spinal cord.

\section{[H2] Transdermal delivery}

Ultrasound has been widely explored as an alternative to conventional injection for transdermal delivery ${ }^{155,156,157}$. Vaccine delivery typically targets the epidermis, where Langerhans cells [G] induce mechanisms of immune response, whereas drug delivery is normally aimed at the greater blood supply available in the dermis. Early studies typically used $\mathrm{kHz}$-range ultrasound without cavitation nucleation agents ${ }^{158,159}$ and focussed primarily on using cavitation to reversibly permeabilize the upper layer of the skin, the stratum corneum, to improve passive diffusion of a drug or vaccine applied to the skin ${ }^{160,161,162}$. More recently, however, the inclusion of nanoscale cavitation nucleation agents alongside the drug or vaccine formulation has enabled the use of microstreaming to simultaneously permeabilize the skin and actively transport the therapeutic across it ${ }^{163}$ to depths in excess of $400 \mu \mathrm{m}^{164}$. This approach has enabled substantially improved delivery of both micromolecular drugs, such as insulin, ${ }^{165}$ and an immune response caused by vaccines that exceeds that achievable by other transdermal methods ${ }^{164}$ (Fig.4b). Despite these considerable advances, cavitation-enhanced transdermal delivery is not yet able to match the immunization efficiency of conventional approaches. The greatest remaining challenge in using it as a tool for drug delivery is that of dose monitoring and confirmation of delivery, because skin properties vary substantially as a function of ambient temperature and humidity.

\section{[H1] Future Developments and Challenges}

Early clinical trials of cavitation-enhanced drug delivery used microbubble formulations initially developed for diagnostic purposes, not because they were necessarily optimal for the intended therapeutic application, but because of their commercial availability and pre-existing regulatory approval which reduced the time and cost of clinical translation. However, the size, fragility and limited circulation time of microbubbles render them sub-optimal for certain therapeutic applications. For example, the inability of microbubbles to penetrate solid tumour vasculature limits their ability to transport therapeutics compared to sub-micrometre scale agents ${ }^{131,132}$. Microbubble destruction and depletion is also rapid in excitation regimes requiring inertial cavitation, including certain types of sonoporation-mediated gene transfer ${ }^{166}$. In applications such as transdermal delivery, the ability of the cavitation nucleation agents to remain intact as they cross the stratum corneum is similarly proving important in mediating improved penetration of drugs ${ }^{164}$.

Thus the development and clinical translation of application-specific cavitation nucleation agents that provide the appropriate size, circulation characteristics and post-excitation cavitation dynamics presents a major opportunity for future research (Fig. 5a,b). For nanodroplets in particular, there is also a need to better understand the relationship between particle characteristics and optimal 
exposure conditions. There is still only limited understanding of the physical and biochemical interactions between cavitation agents and the immune system, endothelium, tissue matrix and cellular and intracellular components. With the notable advances in immunotherapy over the past decade, this is an area requiring development. Moreover, in many cases, these next-generation agents exploit not only responsiveness to ultrasound but to other stimuli, such as magnetic fields, to improve retention and delivery performance ${ }^{167}$.

Clinical studies in recent years have also outlined the need for improved methods of real-time detection, localization, classification and quantification of cavitation activity, which cannot be addressed by either conventional B-mode ultrasound or by magnetic resonance imaging. A decade after its inception ${ }^{113}$, PAM using conventional diagnostic ultrasound has evolved from its initial timedomain ${ }^{114,118}$ and frequency-domain ${ }^{115,168}$ implementations to more robust algorithms that improve spatial resolution and are better suited to integration and co-registration with existing ultrasound-based or MRI-based treatment guidance techniques in a clinical environment ${ }^{121,169}$ (Fig.5c,d).

Two research areas arise from this improved ability to map acoustic cavitation in real time. First, because PAM enables quantification and characterization of cavitation activity at multiple locations simultaneously, existing strategies for the control of ultrasound exposure conditions based on a single scalar metric of cavitation activity ${ }^{170,171}$ must evolve to enable 2D or even 3D optimization of cavitation activity ${ }^{137}$ (Fig.5e). Second, PAM offers a unique opportunity to correlate acoustic emissions spatio-temporally with specific bioeffects (Fig. 5d). This correlation will enable better understanding of the detailed mechanisms of permeabilization, transport and cell death, ultimately facilitating regulatory approval and further clinical exploration of innovative cavitation-based therapies.

Finally, we believe that a common mistake of the past has been to combine cutting-edge drug delivery technologies with dated therapeutics that are perceived as safe. Ultimately, physically actuated remote-control drug delivery will be most transformative when combined with highly specific, self-amplifying modern therapeutics that harness the cellular, extracellular and immune environments to best exploit the improved local distribution, reduced toxicity and enhanced therapeutic index afforded by cavitation. 


\section{References}

1. Lohse D, Schmitz B, Versluis M. Snapping shrimp make flashing bubbles. Nature 2001, 413(6855): 477-478.

2. Suslick KS. Sonochemistry. Science 1990, 247(4949): 1439-1445.

3. Mitragotri S. Innovation - Healing sound: the use of ultrasound in drug delivery and other therapeutic applications. Nature Reviews Drug Discovery 2005, 4(3): 255-260.

4. Lehmann JF, Herrick JF. Biologic reactions to cavitation, a consideration for ultrasonic therapy. Arch Phys Med Rehabil 1953, 34(2): 86-98.

5. Nyborg WL. Biological effects of ultrasound: Development of safety guidelines. Part II: General review. Ultrasound Med Biol 2001, 27(3): 301-333.

6. Crum LA. Cavitation microjets as a contributory mechanism for renal calculi disintegration in ESWL. J Urol 1988, 140(6): 1587-1590.

7. Kennedy JE. High-intensity focused ultrasound in the treatment of solid tumours. Nature Reviews Cancer 2005, 5(4): 321-327.

8. Fechheimer M, Boylan JF, Parker S, Sisken JE, Patel GL, Zimmer SG. Transfection of Mammalian-Cells with Plasmid DNA by Scrape Loading and Sonication Loading. P Natl Acad Sci USA 1987, 84(23): 8463-8467.

9. McDannold N, Vykhodtseva N, Hynynen K. Targeted disruption of the blood-brain barrier with focused ultrasound: association with cavitation activity. Phys Med Biol 2006, 51(4): 793807.

10. Langer R. Drug delivery and targeting. Nature 1998, 392(6679 Suppl): 5-10.

11. Husseini GA, Pitt WG. Micelles and nanoparticles for ultrasonic drug and gene delivery. Adv Drug Deliv Rev 2008, 60(10): 1137-1152.

12. Evjen TJ, Hagtvet E, Moussatov A, Røgnvaldsson S, Mestas J-L, Fowler RA, et al. In vivo monitoring of liposomal release in tumours following ultrasound stimulation. European Journal of Pharmaceutics and Biopharmaceutics 2013, 84(3): 526-531.

13. Graham SM, Carlisle R, Choi JJ, Stevenson M, Shah AR, Myers RS, et al. Inertial cavitation to non-invasively trigger and monitor intratumoral release of drug from intravenously delivered liposomes. Journal of Controlled Release 2014, 178: 101-107. 
14. Kolb J, Nyborg WL. Small-Scale Acoustic Streaming in Liquids. J Acoust Soc Am 1956, 28(6): 1237-1242.

15. Nyborg WL. Acoustic Streaming near a Boundary. J Acoust Soc Am 1958, 30(4): 329-339.

16. Elder S, Nyborg WL. Acoustic Streaming Resulting from a Resonant Bubble. J Acoust Soc Am 1956, 28(1): 155-155.

17. Marmottant $\mathrm{P}$, Hilgenfeldt $\mathrm{S}$. Controlled vesicle deformation and lysis by single oscillating bubbles. Nature 2003, 423(6936): 153-156.

18. Pereno VC, D.;Stride, E. Cavitation induced intracellular streaming. (under review) 2018.

19. A NCE. On the Mechanism of Cavitation Damage by Nonhemispherical Cavities Collapsing in Contact With a Solid Boundary. Journal of Basic Engineering 1961, 83(4): 648-656.

20. Benjamin TE, A. The Collapse of Cavitation Bubbles and the Pressures thereby Produced against Solid Boundaries. Philosophical Transactions of the Royal Society of London Series A, Mathematical and Physical Sciences, 260, (1110): 221-240.

21. Enayati $\mathrm{M}, \mathrm{Al}$ Mohazey $\mathrm{D}$, Edirisinghe $\mathrm{M}$, Stride $\mathrm{E}$. Ultrasound-stimulated drug release from polymer micro and nanoparticles. Bioinspir Biomim Nan 2013, 2(1): 3-10.

22. Ahmed SE, Martins AM, Husseini GA. The use of ultrasound to release chemotherapeutic drugs from micelles and liposomes. J Drug Target 2015, 23(1): 16-42.

23. Hilgenfeldt $S$, Lohse $D$, Zomack $M$. Response of bubbles to diagnostic ultrasound: a unifying theoretical approach. Eur Phys J B 1998, 4(2): 247-255.

24. Holt RG, Roy RA. Measurements of bubble-enhanced heating from focused, MHz-frequency ultrasound in a tissue-mimicking material. Ultrasound Med Biol 2001, 27(10): 1399-1412.

25. Hilgenfeldt $S$, Lohse $D$. The acoustics of diagnostic microbubbles: dissipative effects and heat deposition. Ultrasonics 2000, 38(1-8): 99-104.

26. Hilgenfeldt S, Lohse $D$, Zomack M. Sound scattering and localized heat deposition of pulsedriven microbubbles. J Acoust Soc Am 2000, 107(6): 3530-3539.

27. Yudina A, de Smet $M$, Lepetit-Coiffe $M$, Langereis $S$, Van Ruijssevelt L, Smirnov $P$, et al. Ultrasound-mediated intracellular drug delivery using microbubbles and temperaturesensitive liposomes. J Control Release 2011, 155(3): 442-448. 
28. Coussios CC, Roy RA. Applications of acoustics and cavitation to noninvasive therapy and drug delivery. Annu Rev Fluid Mech 2008, 40: 395-420.

29. Prosperetti A. Thermal Effects and Damping Mechanisms in Forced Radial Oscillations of Gas-Bubbles in Liquids. J Acoust Soc Am 1977, 61(1): 17-27.

30. Flint EB, Suslick KS. The temperature of cavitation. Science 1991, 253(5026): 1397-1399.

31. Winterbourn CC. Reconciling the chemistry and biology of reactive oxygen species. Nat Chem Biol 2008, 4(5): 278-286.

32. McEwan C, Kamila S, Owen J, Nesbitt H, Callan B, Borden M, et al. Combined sonodynamic and antimetabolite therapy for the improved treatment of pancreatic cancer using oxygen loaded microbubbles as a delivery vehicle. Biomaterials 2016, 80: 20-32.

33. Lee JY, Crake C, Teo B, Carugo D, de Saint Victor M, Seth A, et al. Ultrasound-Enhanced siRNA Delivery Using Magnetic Nanoparticle-Loaded Chitosan-Deoxycholic Acid Nanodroplets. Adv Healthc Mater 2017, 6(8).

34. Rosenthal I, Sostaric JZ, Riesz P. Sonodynamic therapy--a review of the synergistic effects of drugs and ultrasound. Ultrason Sonochem 2004, 11(6): 349-363.

35. Bohmer MR, Chlon CHT, Raju BI, Chin CT, Shevchenko T, Klibanov AL. Focused ultrasound and microbubbles for enhanced extravasation. J Control Release 2010, 148(1): 18-24.

36. Carlisle R, Coussios C-C. Mechanical approaches to oncological drug delivery. Therapeutic delivery 2013, 4(10): 1213-1215.

37. Arvanitis CD, Bazan-Peregrino M, Rifai B, Seymour LW, Coussios CC. Cavitation-enhanced extravasation for drug delivery. Ultrasound Med Biol 2011, 37(11): 1838-1852.

38. Carlisle R, Choi J, Bazan-Peregrino M, Laga R, Subr V, Kostka L, et al. Enhanced Tumor Uptake and Penetration of Virotherapy Using Polymer Stealthing and Focused Ultrasound. Jnci-J Natl Cancer / 2013, 105(22): 1701-1710.

39. Rifai B, Arvanitis CD, Bazan-Peregrino M, Coussios CC. Cavitation-enhanced delivery of macromolecules into an obstructed vessel. J Acoust Soc Am 2010, 128(5): El310-El315.

40. van Wamel A, Kooiman K, Harteveld M, Emmer M, ten Cate FJ, Versluis M, et al. Vibrating microbubbles poking individual cells: drug transfer into cells via sonoporation. J Control Release 2006, 112(2): 149-155. 
41. Kudo N. High-Speed In Situ Observation System for Sonoporation of Cells With Size- and Position-Controlled Microbubbles. IEEE Trans Ultrason Ferroelectr Freq Control 2017, 64(1): 273-280.

42. Abbott NJ. Blood-brain barrier structure and function and the challenges for CNS drug delivery. J Inherit Metab Dis 2013, 36(3): 437-449.

43. Jia C, Xu L, Han T, Cai P, Yu ACH, Qin P. Generation of Reactive Oxygen Species in Heterogeneously Sonoporated Cells by Microbubbles with Single-Pulse Ultrasound. Ultrasound Med Biol 2018, 44(5): 1074-1085.

44. Kooiman $\mathrm{K}$, van der Steen AF, de Jong N. Role of intracellular calcium and reactive oxygen species in microbubble-mediated alterations of endothelial layer permeability. IEEE Trans Ultrason Ferroelectr Freq Control 2013, 60(9): 1811-1815.

45. Juffermans LJ, Kamp O, Dijkmans PA, Visser CA, Musters RJ. Low-intensity ultrasoundexposed microbubbles provoke local hyperpolarization of the cell membrane via activation of BK(Ca) channels. Ultrasound Med Biol 2008, 34(3): 502-508.

46. Helfield BL, Chen XC, Qin B, Watkins SC, Villanueva FS. Mechanistic Insight into Sonoporation with Ultrasound-Stimulated Polymer Microbubbles. Ultrasound Med Biol 2017, 43(11): 26782689.

47. Acconcia CN, Leung BY, Goertz DE. The microscale evolution of the erosion front of blood clots exposed to ultrasound stimulated microbubbles. J Acoust Soc Am 2016, 139(5): EL135.

48. Bader KB, Gruber MJ, Holland CK. Shaken and stirred: mechanisms of ultrasound-enhanced thrombolysis. Ultrasound Med Biol 2015, 41(1): 187-196.

49. Miller DL, Thomas RM, Williams AR. Mechanisms for hemolysis by ultrasonic cavitation in the rotating exposure system. Ultrasound Med Biol 1991, 17(2): 171-178.

50. Caskey CF, Qin S, Dayton PA, Ferrara KW. Microbubble tunneling in gel phantoms. J Acoust Soc Am 2009, 125(5): EL183-189.

51. Samiotaki G, Konofagou EE. Dependence of the reversibility of focused- ultrasound-induced blood-brain barrier opening on pressure and pulse length in vivo. IEEE Trans Ultrason Ferroelectr Freq Control 2013, 60(11): 2257-2265.

52. Plesset MS, Prosperetti A. Bubble Dynamics and Cavitation. Annu Rev Fluid Mech 1977, 9: 145-185. 
53. Lajoinie G, Luan Y, Gelderblom E, Dollet B, Mastik F, Dewitte H, et al. Non-spherical oscillations drive the ultrasound-mediated release from targeted microbubbles. Commun Phys 2018, 1.

54. Kudo N, Kinoshita Y. Effects of cell culture scaffold stiffness on cell membrane damage induced by sonoporation. J Med Ultrason (2001) 2014, 41(4): 411-420.

55. Chen H, Brayman AA, Kreider W, Bailey MR, Matula TJ. Observations of translation and jetting of ultrasound-activated microbubbles in mesenteric microvessels. Ultrasound Med Biol 2011, 37(12): 2139-2148.

56. Martynov S, Kostson E, Saffari N, Stride E. Forced vibrations of a bubble in a liquid-filled elastic vessel. J Acoust Soc Am 2011, 130(5): 2700-2708.

57. Chen X, Wang J, Pacella JJ, Villanueva FS. Dynamic Behavior of Microbubbles during Long Ultrasound Tone-Burst Excitation: Mechanistic Insights into Ultrasound-Microbubble Mediated Therapeutics Using High-Speed Imaging and Cavitation Detection. Ultrasound Med Biol 2016, 42(2): 528-538.

58. Lentacker I, De Cock I, Deckers R, De Smedt SC, Moonen CT. Understanding ultrasound induced sonoporation: definitions and underlying mechanisms. Adv Drug Deliv Rev 2014, 72: 49-64.

59. Qin P, Han T, Yu ACH, Xu L. Mechanistic understanding the bioeffects of ultrasound-driven microbubbles to enhance macromolecule delivery. J Control Release 2018, 272: 169-181.

60. W. HEBDM. Bubble formation in animals. J Cellular and Comparative Physiology 1944, 24: 122.

61. Briggs L. Limiting Negative Pressure of Water. J Appl Phys 1950, 21(7): 721-722.

62. Morch KA. Cavitation inception from bubble nuclei. Interface Focus 2015, 5(5).

63. Strasberg M. Onset of Ultrasonic Cavitation in Tap Water. J Acoust Soc Am 1959, 31(2): 163176.

64. Atchley AA, Prosperetti A. The Crevice Model of Bubble Nucleation. J Acoust Soc Am 1989, 86(3): 1065-1084.

65. Borkent BM, Gekle S, Prosperetti A, Lohse D. Nucleation threshold and deactivation mechanisms of nanoscopic cavitation nuclei. Phys Fluids 2009, 21(10). 
66. Fox FE, Herzfeld KF. Gas Bubbles with Organic Skin as Cavitation Nuclei. J Acoust Soc Am 1954, 26(6): 984-989.

67. Yount DE. Skins of Varying Permeability - Stabilization Mechanism for Gas Cavitation Nuclei. J Acoust Soc Am 1979, 65(6): 1429-1439.

68. Blake FG. Technical Memo. 12, Acoustics Research Laboratory, Harvard University, . 6. . 1949.

69. Hsieh DY, Plesset MS. Theory of Rectified Diffusion of Mass into Gas Bubbles. J Acoust Soc Am 1961, 33(2): 206-\&.

70. Church CC. The Effects of an Elastic Solid-Surface Layer on the Radial Pulsations of GasBubbles. J Acoust Soc Am 1995, 97(3): 1510-1521.

71. Carugo D, Aron M, Sezgin E, de la Serna JB, Kuirnova MK, Eggeling C, et al. Modulation of the molecular arrangement in artificial and biological membranes by phospholipid-shelled microbubbles. Biomaterials 2017, 113: 105-117.

72. Lentacker I, De Smedt SC, Sanders NN. Drug loaded microbubble design for ultrasound triggered delivery. Soft Matter 2009, 5(11): 2161-2170.

73. Mulvana H, Browning RJ, Luan Y, de Jong N, Tang MX, Eckersley RJ, et al. Characterization of Contrast Agent Microbubbles for Ultrasound Imaging and Therapy Research. leee T Ultrason Ferr 2017, 64(1): 232-251.

74. Tinkov S, Coester C, Serba S, Geis NA, Katus HA, Winter G, et al. New doxorubicin-loaded phospholipid microbubbles for targeted tumor therapy: In-vivo characterization. Journal of Controlled Release 2010, 148(3): 368-372.

75. Geers B, Lentacker I, Sanders NN, Demeester J, Meairs S, De Smedt SC. Self-assembled liposome-loaded microbubbles: The missing link for safe and efficient ultrasound triggered drug-delivery. Journal of Controlled Release 2011, 152(2): 249-256.

76. Geers B, Dewitte H, De Smedt SC, Lentacker I. Crucial factors and emerging concepts in ultrasound-triggered drug delivery. Journal of Controlled Release 2012, 164(3): 248-255.

77. Vlaskou D, Mykhaylyk O, Pradhan P, Bergemann C, Klibanov AL, Hensel K, et al. Magnetic Microbubbles: Magnetically Targeted and Ultrasound-Triggered Vectors for Gene Delivery In Vitro. Hum Gene Ther 2010, 21(9): 1191-1191. 
78. Sheng YJ, Beguin E, Nesbitt H, Kamila S, Owen J, Barnsley LC, et al. Magnetically responsive microbubbles as delivery vehicles for targeted sonodynamic and antimetabolite therapy of pancreatic cancer. Journal of Controlled Release 2017, 262: 192-200.

79. McEwan C, Owen J, Stride E, Fowley C, Nesbitt H, Cochrane D, et al. Oxygen carrying microbubbles for enhanced sonodynamic therapy of hypoxic tumours. Journal of Controlled Release 2015, 203: 51-56.

80. Grishenkov D, Gonon A, Weitzberg E, Lundberg JO, Harmark J, Cerroni B, et al. Ultrasound contrast agent loaded with nitric oxide as a theranostic microdevice. Drug Des Devel Ther 2015, 9: 2409-2419.

81. Morel DR, Schwieger I, Hohn L, Terrettaz J, Llull JB, Cornioley YA, et al. Human pharmacokinetics and safety evaluation of SonoVue, a new contrast agent for ultrasound imaging. Invest Radiol 2000, 35(1): 80-85.

82. Rapoport N, Gao Z, Kennedy A. Multifunctional nanoparticles for combining ultrasonic tumor imaging and targeted chemotherapy. J Natl Cancer Inst 2007, 99(14): 1095-1106.

83. Wilhelm S, Tavares AJ, Dai Q, Ohta S, Audet J, Dvorak HF, et al. Analysis of nanoparticle delivery to tumours. Nat Rev Mater 2016, 1(5).

84. Sheeran PS, Luois S, Dayton PA, Matsunaga TO. Formulation and Acoustic Studies of a New Phase-Shift Agent for Diagnostic and Therapeutic Ultrasound. Langmuir 2011, 27(17): 1041210420.

85. Javadi M, Pitt WG, Belnap DM, Tsosie NH, Hartley JM. Encapsulating Nanoemulsions Inside eliposomes for Ultrasonic Drug Delivery. Langmuir 2012, 28(41): 14720-14729.

86. Wang $\mathrm{CH}$, Kang ST, Lee YH, Luo YL, Huang YF, Yeh CK. Aptamer-conjugated and drug-loaded acoustic droplets for ultrasound theranosis. Biomaterials 2012, 33(6): 1939-1947.

87. Yu JS, Chen ZY, Li Y, Du M, Yan F, Zheng HR. Echogenic Chitosan Nanodroplets for Spatiotemporally Controlled Gene Delivery. J Biomed Nanotechnol 2018, 14(7): 1287-1297.

88. Moyer LC, Timbie KF, Sheeran PS, Price RJ, Miller GW, Dayton PA. High-intensity focused ultrasound ablation enhancement in vivo via phase-shift nanodroplets compared to microbubbles. J Ther Ultrasound 2015, 3: 7.

89. Ho YJ, Yeh CK. Theranostic Performance of Acoustic Nanodroplet Vaporization-Generated Bubbles in Tumor Intertissue. Theranostics 2017, 7(6): 1477-1488. 
90. Chen CC, Sheeran PS, Wu SY, Olumolade OO, Dayton PA, Konofagou EE. Targeted drug delivery with focused ultrasound-induced blood-brain barrier opening using acousticallyactivated nanodroplets. Journal of Controlled Release 2013, 172(3): 795-804.

91. Sheeran PS, Matsunaga TO, Dayton PA. Phase-transition thresholds and vaporization phenomena for ultrasound phase-change nanoemulsions assessed via high-speed optical microscopy. Physics in Medicine and Biology 2013, 58(13): 4513-4534.

92. Shpak O, Verweij M, Vos HJ, de Jong $\mathrm{N}$, Lohse $\mathrm{D}$, Versluis $\mathrm{M}$. Acoustic droplet vaporization is initiated by superharmonic focusing. P Natl Acad Sci USA 2014, 111(5): 1697-1702.

93. Wang Y, Liu G, Hu H, Li TY, Johri AM, Li X, et al. Stable Encapsulated Air Nanobubbles in Water. Angew Chem Int Ed Eng/ 2015, 54(48): 14291-14294.

94. Hernandez C, Nieves L, de Leon AC, Advincula R, Exner AA. Role of Surface Tension in Gas Nanobubble Stability Under Ultrasound. ACS Appl Mater Interfaces 2018, 10(12): 9949-9956.

95. Paris JL, Mannaris C, Cabanas MV, Carlisle R, Manzano M, Vallet-Regi M, et al. Ultrasoundmediated cavitation-enhanced extravasation of mesoporous silica nanoparticles for controlled-release drug delivery. Chem Eng J 2018, 340: 2-8.

96. Delogu LG, Vidili G, Venturelli E, Menard-Moyon C, Zoroddu MA, Pilo G, et al. Functionalized multiwalled carbon nanotubes as ultrasound contrast agents. Proc Natl Acad Sci U S A 2012, 109(41): 16612-16617.

97. Straub JA, Chickering DE, Church CC, Shah B, Hanlon T, Bernstein H. Porous PLGA microparticles: Al-700, an intravenously administered ultrasound contrast agent for use in echocardiography. J Control Release 2005, 108(1): 21-32.

98. Kwan J, Graham S, Myers R, Carlisle R, Stride E, Coussios C. Ultrasound-induced inertial cavitation from gas-stabilizing nanoparticles. Physical Review E 2015, 92(2): 023019.

99. Mannaris C, Teo BM, Seth A, Bau L, Coussios C, Stride E. Gas - Stabilizing Gold Nanocones for Acoustically Mediated Drug Delivery. Advanced healthcare materials 2018: 1800184.

100. Kang E, Min HS, Lee J, Han MH, Ahn HJ, Yoon IC, et al. Nanobubbles from gas-generating polymeric nanoparticles: ultrasound imaging of living subjects. Angew Chem Int Ed Engl 2010, 49(3): 524-528.

101. Toyokuni S. Genotoxicity and carcinogenicity risk of carbon nanotubes. Adv Drug Deliv Rev 2013, 65(15): 2098-2110. 
102. Prosperetti A, Crum LA, Commander KW. Nonlinear Bubble Dynamics. J Acoust Soc Am 1988, 83(2): 502-514.

103. Flynn HG. Cavitation Dynamics .2. Free Pulsations and Models for Cavitation Bubbles. J Acoust Soc Am 1975, 58(6): 1160-1170.

104. Church CC, Carstensen EL. "Stable" inertial cavitation. Ultrasound Med Biol 2001, 27(10): 1435-1437.

105. Neppiras EA. Acoustic Cavitation. Phys Rep 1980, 61(3): 159-251.

106. Apfel RE, Holland CK. Gauging the Likelihood of Cavitation from Short-Pulse, Low-Duty Cycle Diagnostic Ultrasound. Ultrasound Med Biol 1991, 17(2): 179-185.

107. Madanshetty SI, Roy R, Apfel RE. Acoustic microcavitation: its active and passive acoustic detection. J Acoust Soc Am 1991, 90(3): 1515-1526.

108. Rabkin BA, Zderic V, Vaezy S. Hyperecho in ultrasound images of HIFU therapy: Involvement of cavitation. Ultrasound in Medicine and Biology 2005, 31(7): 947-956.

109. Coussios CC, Farny $\mathrm{CH}$, Haar GT, Roy RA. Role of acoustic cavitation in the delivery and monitoring of cancer treatment by high-intensity focused ultrasound (HIFU). Int J Hyperthermia 2007, 23(2): 105-120.

110. Arnal B, Baranger J, Demene C, Tanter M, Pernot M. In vivo real-time cavitation imaging in moving organs. Physics in Medicine \& Biology 2017, 62(3): 843.

111. Gateau J, Aubry J-F, Pernot M, Fink M, Tanter M. Combined passive detection and ultrafast active imaging of cavitation events induced by short pulses of high-intensity ultrasound. IEEE transactions on ultrasonics, ferroelectrics, and frequency control 2011, 58(3): 517-532.

112. Rabkin BA, Zderic V, Crum LA, Vaezy S. Biological and physical mechanisms of HIFU-induced hyperecho in ultrasound images. Ultrasound in Medicine \& Biology 2006, 32(11): 1721-1729.

113. Gyongy M, Arora M, Noble JA, Coussios CC. Use of passive arrays for characterization and mapping of cavitation activity during HIFU exposure. Ultrasonics Symposium, 2008. IUS 2008. IEEE; 2008: IEEE; 2008. p. 871-874.

114. Gyongy M, Coussios CC. Passive spatial mapping of inertial cavitation during HIFU exposure. Biomedical Engineering, IEEE Transactions on 2010, 57(1): 48-56. 
115. Salgaonkar VA, Datta S, Holland CK, Mast TD. Passive cavitation imaging with ultrasound arrays. The Journal of the Acoustical Society of America 2009, 126(6): 3071-3083.

116. Haworth KJ, Mast TD, Radhakrishnan K, Burgess MT, Kopechek JA, Huang S-L, et al. Passive imaging with pulsed ultrasound insonations. The Journal of the Acoustical Society of America 2012, 132: 544 .

117. Arvanitis CD, Crake C, McDannold N, Clement GT. Passive Acoustic Mapping with the Angular Spectrum Method. leee T Med Imaging 2017, 36(4): 983-993.

118. Gyongy M, Coussios CC. Passive cavitation mapping for localization and tracking of bubble dynamics. Journal of the Acoustical Society of America 2010, 128(4): E175-E180.

119. Jensen CR, Ritchie RW, Gyongy M, Collin JRT, Leslie T, Coussios CC. Spatiotemporal Monitoring of High-Intensity Focused Ultrasound Therapy with Passive Acoustic Mapping. Radiology 2012, 262(1): 252-261.

120. Gray MD, Lyka E, Coussios CC. Diffraction Effects and Compensation in Passive Acoustic Mapping. leee T Ultrason Ferr 2018, 65(2): 258-268.

121. Coviello C, Kozick R, Choi J, Gyöngy M, Jensen C, Smith PP, et al. Passive acoustic mapping utilizing optimal beamforming in ultrasound therapy monitoring. The Journal of the Acoustical Society of America 2015, 137(5): 2573-2585.

122. Choi JJ, Carlisle RC, Coviello C, Seymour L, Coussios C-C. Non-invasive and real-time passive acoustic mapping of ultrasound-mediated drug delivery. Physics in medicine and biology 2014, 59(17): 4861.

123. Kwan JJ, Myers R, Coviello CM, Graham SM, Shah AR, Stride E, et al. Ultrasound-Propelled Nanocups for Drug Delivery. Small 2015, 11(39): 5305-5314.

124. Miller DL. Overview of experimental studies of biological effects of medical ultrasound caused by gas body activation and inertial cavitation. Progress in biophysics and molecular biology 2007, 93(1-3): 314-330.

125. Hobbs SK, Monsky WL, Yuan F, Roberts WG, Griffith L, Torchilin VP, et al. Regulation of transport pathways in tumor vessels: role of tumor type and microenvironment. Proceedings of the National Academy of Sciences 1998, 95(8): 4607-4612.

126. Bazan-Peregrino M, Arvanitis CD, Rifai B, Seymour LW, Coussios CC. Ultrasound-induced cavitation enhances the delivery and therapeutic efficacy of an oncolytic virus in an in vitro model. J Control Release 2012, 157(2): 235-242. 
127. Bazan-Peregrino M, Rifai B, Carlisle RC, Choi J, Arvanitis CD, Seymour LW, et al. Cavitationenhanced delivery of a replicating oncolytic adenovirus to tumors using focused ultrasound. J Control Release 2013, 169(1-2): 40-47.

128. Lafond M, Aptel F, Mestas JL, Lafon C. Ultrasound-mediated ocular delivery of therapeutic agents: a review. Expert Opin Drug Del 2017, 14(4): 539-550.

129. Prieur F, Pillon A, Mestas JL, Cartron V, Cebe P, Chansard N, et al. Enhancement of Fluorescent Probe Penetration into Tumors in Vivo Using Unseeded Inertial Cavitation. Ultrasound Med Biol 2016, 42(7): 1706-1713.

130. Li T, Wang Y-N, Khokhlova TD, D'Andrea S, Starr F, Chen H, et al. Pulsed high intensity focused ultrasound ( $\mathrm{pHIFU}$ ) enhances delivery of doxorubicin in a preclinical model of pancreatic cancer. Cancer research 2015: canres. 0296.2015.

131. Myers R, Coviello C, Erbs P, Foloppe J, Rowe C, Kwan J, et al. Polymeric Cups for Cavitationmediated Delivery of Oncolytic Vaccinia Virus. Mol Ther 2016, 24(9): 1627-1633.

132. Dimcevski G, Kotopoulis S, Bjånes T, Hoem D, Schjøtt J, Gjertsen BT, et al. A human clinical trial using ultrasound and microbubbles to enhance gemcitabine treatment of inoperable pancreatic cancer. Journal of Controlled Release 2016, 243: 172-181.

133. Hynynen K, McDannold N, Vykhodtseva N, Jolesz FA. Noninvasive MR imaging-guided focal opening of the blood-brain barrier in rabbits. Radiology 2001, 220(3): 640-646.

134. McDannold N, Vykhodtseva N, Hynynen K. Targeted disruption of the blood-brain barrier with focused ultrasound: association with cavitation activity. Physics in Medicine \& Biology 2006, 51(4): 793.

135. Tung Y-S, Vlachos F, Choi JJ, Deffieux T, Selert K, Konofagou EE. In vivo transcranial cavitation threshold detection during ultrasound-induced blood-brain barrier opening in mice. Physics in Medicine \& Biology 2010, 55(20): 6141.

136. Arvanitis $C D$, Livingstone MS, McDannold N. Combined ultrasound and MR imaging to guide focused ultrasound therapies in the brain. Physics in Medicine and Biology 2013, 58(14): 4749-4761.

137. Jones RM, Deng LL, Leung K, McMahon D, O'Reilly MA, Hynynen K. Three-dimensional transcranial microbubble imaging for guiding volumetric ultrasound-mediated blood-brain barrier opening. Theranostics 2018, 8(11): 2909-2926.

138. Carpentier A, Canney M, Vignot A, Reina V, Beccaria K, Horodyckid C, et al. Clinical trial of blood-brain barrier disruption by pulsed ultrasound. Sci Transl Med 2016, 8(343): 343re342. 
139. Lipsman N, Meng Y, Bethune AJ, Huang Y, Lam B, Masellis M, et al. Blood-brain barrier opening in Alzheimer's disease using MR-guided focused ultrasound. Nature communications 2018, 9(1): 2336.

140. de Saint Victor M, Crake C, Coussios C-C, Stride E. Properties, characteristics and applications of microbubbles for sonothrombolysis. Expert opinion on drug delivery 2014, 11(2): 187-209.

141. Mercado-Shekhar KP, Kleven RT, Rivera HA, Lewis R, Karani KB, Vos HJ, et al. Effect of Clot Stiffness on Recombinant Tissue Plasminogen Activator Lytic Susceptibility in Vitro. Ultrasound in medicine \& biology 2018.

142. Datta S, Coussios C, Ammi A, Mast T, de Courten-Myers G, Holland C. Ultrasound-enhanced thrombolysis using Definity ${ }^{\circledR}$ as a cavitation nucleation agent. Ultrasound in Medicine \& Biology 2008, 34(9): 1421-1433.

143. Datta S, Coussios CC, McAdory LE, Tan J, Porter T, De Courten-Myers G, et al. Correlation of cavitation with ultrasound enhancement of thrombolysis. Ultrasound in Medicine and Biology 2006, 32(8): 1257-1267.

144. Bader KB, Gruber MJ, Holland CK. Shaken and stirred: mechanisms of ultrasound-enhanced thrombolysis. Ultrasound in medicine \& biology 2015, 41(1): 187-196.

145. Molina CA, Ribo M, Rubiera M, Montaner J, Santamarina E, Delgado-Mederos R, et al. Microbubble administration accelerates clot lysis during continuous 2-MHz ultrasound monitoring in stroke patients treated with intravenous tissue plasminogen activator. Stroke 2006, 37(2): 425-429.

146. Mathias W, Tavares BG, Aguiar MO, Tsutsui J, Chiang H, Soeiro A, et al. THE EFFECTIVENESS OF MICROBUBBLE-MEDIATED SONOTHROMBOLYSIS FOR INDUCING EARLY RECANALIZATION OF DIFFERENT CULPRIT CORONARY ARTERIES IN PATIENTS WITH ACUTE ST-SEGMENT ELEVATION MYOCARDIAL INFARCTION. Journal of the American College of Cardiology 2018, 71(11): A1460.

147. Mathias W, Tsutsui JM, Tavares BG, Xie F, Aguiar MO, Garcia DR, et al. Diagnostic ultrasound impulses improve microvascular flow in patients with STEMI receiving intravenous microbubbles. Journal of the American College of Cardiology 2016, 67(21): 2506-2515.

148. Molina CA, Barreto AD, Tsivgoulis G, Sierzenski P, Malkoff MD, Rubiera M, et al. Transcranial ultrasound in clinical sonothrombolysis (TUCSON) trial. Ann Neurol 2009, 66(1): 28-38.

149. Owen J, Rademeyer P, Chung D, Cheng Q, Holroyd D, Coussios C, et al. Magnetic targeting of microbubbles against physiologically relevant flow conditions. Interface focus 2015, 5(5): 20150001. 
150. Vignon F, Shi WT, Powers JE, Everbach EC, Liu J, Gao S, et al. Microbubble cavitation imaging. IEEE transactions on ultrasonics, ferroelectrics, and frequency control 2013, 60(4): 661-670.

151. Haworth KJ, Mast TD, Radhakrishnan K, Burgess MT, Kopechek JA, Huang S-L, et al. Passive imaging with pulsed ultrasound insonations. The Journal of the Acoustical Society of America 2012, 132(1): 544-553.

152. Qiao S, Coussios C, Cleveland R. Characterization of modular arrays for transpinal ultrasound application. The Journal of the Acoustical Society of America 2017, 141(5): 3954-3954.

153. Fletcher S-MP, O'Reilly MA. Analysis of Multi-Frequency and Phase Keying Strategies for Focusing Ultrasound to the Human Vertebral Canal. IEEE transactions on ultrasonics, ferroelectrics, and frequency control 2018.

154. O’Reilly MA, Chinnery T, Yee M-L, Wu S-K, Hynynen K, Kerbel RS, et al. Preliminary Investigation of Focused Ultrasound-Facilitated Drug Delivery for the Treatment of Leptomeningeal Metastases. Scientific reports 2018, 8(1): 9013.

155. Mitragotri S, Edwards DA, Blankschtein D, Langer R. Mechanistic Study of UltrasonicallyEnhanced Transdermal Drug-Delivery. J Pharm Sci-Us 1995, 84(6): 697-706.

156. Tezel A, Paliwal S, Shen Z, Mitragotri S. Low-frequency ultrasound as a transcutaneous immunization adjuvant. Vaccine 2005, 23(29): 3800-3807.

157. Polat BE, Hart D, Langer R, Blankschtein D. Ultrasound-mediated transdermal drug delivery: Mechanisms, scope, and emerging trends. Journal of Controlled Release 2011, 152(3): 330 348.

158. Mitragotri S, Blankschtein D, Langer R. Transdermal drug delivery using low-frequency sonophoresis. Pharm Res-Dordr 1996, 13(3): 411-420.

159. Tezel A, Sens A, Tuchscherer J, Mitragotri S. Frequency dependence of sonophoresis. Pharm Res-Dordr 2001, 18(12): 1694-1700.

160. Tezel A, Sens A, Mitragotri S. Investigations of the role of cavitation in low-frequency sonophoresis using acoustic spectroscopy. J Pharm Sci-Us 2002, 91(2): 444-453.

161. Tezel A, Mitragotri S. Interactions of inertial cavitation bubbles with stratum corneum lipid bilayers during low-frequency sonophoresis. Biophys J 2003, 85(6): 3502-3512. 
162. Rich KT, Hoerig CL, Rao MB, Mast TD. Relations between acoustic cavitation and skin resistance during intermediate-and high-frequency sonophoresis. Journal of Controlled Release 2014, 194: 266-277.

163. Bhatnagar S, Schiffter H, Coussios CC. Exploitation of Acoustic Cavitation-Induced Microstreaming to Enhance Molecular Transport. J Pharm Sci-Us 2014, 103(6): 1903-1912.

164. Bhatnagar S, Kwan JJ, Shah AR, Coussios CC, Carlisle RC. Exploitation of sub-micron cavitation nuclei to enhance ultrasound-mediated transdermal transport and penetration of vaccines. Journal of Controlled Release 2016, 238: 22-30.

165. Feiszthuber H, Bhatnagar S, Gyöngy M, Coussios C-C. Cavitation-enhanced delivery of insulin in agar and porcine models of human skin. Physics in medicine and biology 2015, 60(6): 2421-2434.

166. Tran DM, Harrang J, Song S, Chen J, Smith BM, Miao CH. Prolonging pulse duration in ultrasound-mediated gene delivery lowers acoustic pressure threshold for efficient gene transfer to cells and small animals. Journal of Controlled Release 2018, 279: 345-354.

167. Lee JY, Carugo D, Crake C, Owen J, de St Victor M, Seth A, et al. Nanoparticle-Loaded ProteinPolymer Nanodroplets for Improved Stability and Conversion Efficiency in Ultrasound Imaging and Drug Delivery. Advanced Materials 2015, 27(37): 5484-5492.

168. Haworth KJ, Bader KB, Rich KT, Holland CK, Mast TD. Quantitative frequency-domain passive cavitation imaging. IEEE transactions on ultrasonics, ferroelectrics, and frequency control 2017, 64(1): 177-191.

169. Lu S, Hu H, Yu X, Long J, Jing B, Zong Y, et al. Passive acoustic mapping of cavitation using eigenspace-based robust Capon beamformer in ultrasound therapy. Ultrasonics sonochemistry 2018, 41: 670-679.

170. Hockham N, Coussios CC, Arora M. A Real-Time Controller for Sustaining Thermally Relevant Acoustic Cavitation During Ultrasound Therapy. leee T Ultrason Ferr 2010, 57(12): 26852694.

171. O'Reilly MA, Hynynen K. Blood-brain barrier: real-time feedback-controlled focused ultrasound disruption by using an acoustic emissions-based controller. Radiology 2012, 263(1): 96-106.

172. Deng L, O'Reilly MA, Jones RM, An R, Hynynen K. A multi-frequency sparse hemispherical ultrasound phased array for microbubble-mediated transcranial therapy and simultaneous cavitation mapping. Physics in Medicine \& Biology 2016, 61(24): 8476. 


\section{Acknowledgements}

The authors thank The Engineering and Physical Sciences Research Council for supporting their work through grants EP/ EP/L024012/1 and EP/L024012.

\section{Author contributions}

All authors researched data for the article, discussed the content, wrote the manuscript and reviewed and edited it before submission.

\section{Competing interests}

Constantin Coussios is a named inventor on several patents pertaining to cavitation nucleation, mapping, monitoring and control, and a founder, director, shareholder and consultant receiving consultancy income from OxSonics Ltd, a spin-out from the University of Oxford developing a commercial product to enable the clinical translation of cavitation-mediated drug delivery. Eleanor Stride is a named inventor on two patents relating to the use of microbubbles for therapeutic applications and a founder of SonoTarg Ltd. a spin-out company developing oxygen loaded microbubbles for cancer treatment

\section{Publisher's note}

Springer Nature remains neutral with regard to jurisdictional claims in published maps and institutional affiliations. 


\section{List of Figures}

Fig. 1. Schematic of barriers to drug delivery from the tissue to the intracellular scale. The size of therapeutics, such as antibodies, oligonucleotides, viruses and liposomes, is greater than that of small-molecule drugs such as conventional chemotherapeutics. The large particle size increases the likelihood of clearance and degradation, by the immune system (phagocytosis), or occurring in the liver (as hepatic filtration) or the kidneys (as renal filtration). It also restricts the ability of the drug to pass through the blood vessel wall (endothelium) and diffuse through the surrounding (interstitial) tissue. At the cellular level, the cell membrane presents a further barrier to many drugs and, for drugs with specific intracellular targets, transport within the cell interior (cytoplasm) may also be necessary.

Fig. 2. Illustration of key cavitation mediated phenomena exploited in drug delivery. al Oscillating bubbles can heat their surrounding liquid, through acoustic reradiation and viscous friction. This heating can be exploited to release drugs from thermally sensitive particles at a target location. $\mathbf{b}$ | Microstreaming of the liquid surrounding a bubble can produce shear stresses that can also promote drug release from microparticles and nanoparticles, enhance convective transport and permeabilize both tissue and cellular membranes (shear-induced permeability). At higher pressure amplitudes, microjetting, bubble tunnelling and shockwave generation may all contribute to tissue permeabilization and to drug release from particles . $\mathbf{c l}$ The high temperatures and pressures produced in the core of a collapsing bubble may generate reactive oxygen species and electromagnetic radiation (sonoluminescence) that may stimulate a variety of biological processes and may be exploited for local activation of certain drugs, an approach known as sonodynamic therapy.

Fig. 3. Schematic of the different mechanisms of cavitation nucleation. Bubbles may be generated through either homogenous or heterogenous nucleation. For drug delivery applications, bubble formation is almost exclusively the result of heterogenous nucleation. This is due in part to the extremely high pressures required for homogenous nucleation that are far outside the range associated with ultrasound therapy, and also to the presence of gaseous nuclei in tissue. These endogenous nuclei are thought to consist of either microscopic or nanoscopic gas bubbles stabilized by a 'skin' of surfactants ${ }^{66,67}$, or crevices of similar scale on the surfaces of biological structures ${ }^{63,64}$. To improve the reproducibility of cavitation activity and/or to reduce the energy required, artificial (exogenous) nuclei may be introduced into tissue. These may be in the form of gas-entrapping solid nanoparticles ${ }^{95}{ }^{100}$, superheated liquid droplets ${ }^{82}$ that undergo a transition to gas bubbles upon exposure to ultrasound, or stabilized gas bubbles.

Fig. 4. Oncological, brain, cardiovascular and transdermal applications of microbubble-enhanced drug delivery. a| Microbubble-enhanced drug delivery for cancer treatment. Upon application of ultrasound, intratumoural extravasation and transport of a therapeutic virus are enhanced. $\mathbf{b}$ | Evidence of enhanced transdermal delivery and penetration of a model vaccine, ovalbumin (OVA), through intact rodent skin induced by ultrasound responsive nanoparticles, nanocups (NC). c| Early evidence of successful microbubble-mediated reversible opening of the blood-brain barriers in humans. The white dashed rectangles indicate the region of interest in the T1 weighted (T1W; T1 the spin-lattice relaxation time) magnetic resonance images. In this region the appearance of areas of increased image intensity indicate extravasation of a contrast agent and hence opening of the blood brain barrier. $\mathbf{d}$ | Improved microvascular replenishment following microbubble-enhanced sonothrombolysis in human hearts. The ultrasound images are taken before (left panel) and after (right panel) treatment. The arrow indicates the region where blood flow has been restored, indicated by the change from low to normal image intensity between the images. Panel a is adapted 
from Ref. ${ }^{38}$. Panel $\mathbf{b}$ is adapted from Ref. ${ }^{164}$. Panel $\mathbf{c}$ is adapted from Ref. ${ }^{138}$. Panel $\mathbf{d}$ is adapted from Ref. ${ }^{147}$.

Fig. 5. Challenges and future directions for applications of acoustic cavitation. al Sub-micrometre solid-particle cavitation nucleation agents (nanocups) yield improved intratumoural penetration and prolonged cavitation persistence compared to microbubbles currently used clinically as contrast agents (SonoVue). This improvement is evidenced by comparing the amplitude and duration of the passive acoustic mapping (PAM) signal . bl These improvements result in enhanced intratumoural drug delivery compared to passive accumulation, acoustically inactive cups (i-cups) or microbubbles, and yield significant improvements in the delivery of a biologic agent, vaccinia oncolytic virus (VV). PBS: phosphate-buffered saline, SV: SonoVue, US: ultrasound, ${ }^{*} \mathrm{P}<0.05, * * \mathrm{P}<0.01$, ns: nonsignificant $P>0.05$. $c \mid$ Determination of location-specific acoustic emissions by passive acoustic mapping (PAM) applied to three regions of cavitation in an agar gel. The left panel shows the passive source power map. The right panel shows the PAM signal from each region, and for comparison a raw channel signal used to generate the PAM (upper trace). d d Correlation between the type, location and frequency content of cavitation activity resulting in reversible or irreversible opening of the blood-brain-barrier in vivo. The left panels show T1-weighted (T1 the spin-lattice relaxation time) magnetic resonance imaging (MRI) images, the right panels show the corresponding cavitation maps, with the pixel with maximum cavitation activity marked by the + . The regions of increased T1 contrast and regions of increased cavitation (indicated by yellow squares) correspond. The upper panels show non-inertial cavitation, the lower panels show inertial cavitation. el A customized hemispherical multi-frequency sparse array (left panel inset) was used for therapy and PAM. The T1weighted MRI image of the treated brain (left panel) indicates where treatment occurred (white arrow). The right panel shows the frequency spectrum of the beamformed signal at the point of maximum intensity. $\mathbf{f} \mid$ Next-generation array-based systems can spatiotemporally map, classify and control cavitation activity in real time. Panel $\mathbf{a}$ is adapted from Ref. ${ }^{123}$. Panel $\mathbf{b}$ is adapted from Ref. ${ }^{131}$. Panel $\mathbf{c}$ is adapted from Ref. ${ }^{118}$. Panel $\mathbf{d}$ is adapted from Ref. ${ }^{136}$. Panel $\mathbf{e}$ is adapted from Ref. ${ }^{172}$. Panel $\mathbf{f}$ contains images courtesy of Institute of Biomedical Engineering.

\section{Glossary Terms}

Lithotripsy: A medical procedure involving the physical destruction of solid masses such as kidney stones.

High-intensity focused ultrasound: In medicine this refers to ultrasound with intensities typically exceeding $1000 \mathrm{Wcm}^{-2}$ used for thermal ablation of tissue, for example for cancer treatment.

Vascular: Relating to vessels, typically blood vessels.

Endothelium: The layer of cells lining the interior surface of blood or lymphatic vessels.

Microstreaming: The microscale circulation of a viscous fluid produced by an oscillating structure.

Tissue phantoms: Synthetic objects whose physical properties are similar to those of tissue, enabling experiments to be conducted in a realistic environment.

Transcytosis: A process by which material is transported through the interior of a cell by encapsulation within vesicles that are formed on one side of the cell and ejected on the other.

Secondary radiation force: The force generated between two objects as a result of their oscillation, which may be attractive or repulsive. 
Echogenicity: Ability to produce strong echoes - reflections or scattering - of an incident ultrasound field.

Superharmonic focusing: The process by which a small acoustically responsive object acts as a lens focusing the high frequency components of a nonlinearly propagated ultrasound wave.

Extravasation: The leakage of a fluid out of its container; in the context of drug delivery, the transport of material out of the blood stream into the surrounding tissue.

Beamform: To combine signals with suitable delays so as to amplify information coming from the region of interest.

Stromal layer: A region of tissue containing cells that are not part of the specific function of the organ in which they reside. In a tumour, this layer consists of cells that are not themselves malignant but present a dense barrier to the diffusion of drugs.

Erythrocyte: Red blood cell, whose primary function is the transport of oxygen throughout the blood stream.

Ischaemic: Referring to restricted blood supply and hence a shortage of oxygen.

Recanalization: The process of restoring flow to a blocked vessel.

Epicardial: Referring to the membrane constituting the outer layer of the heart.

Langerhans cells: Immune cells present in all layers of the epidermis and stimulated during vaccination. 